\title{
Persistence and the importance of nonreward: Some applications of frustration theory and DMOD
}

\author{
HELEN B. DALY and JOHN T. DALY \\ State University of New York, Oswego, New York
}

\begin{abstract}
Unfortunately our world does not always reward us when we expect it, and we must learn to deal with nonreward. How do these experiences influence our behaviors and how can we use them to help us? In Frustration Theory: An Analysis of Dispositional Learning and Memory (1992), Abram Amsel has answered these questions; he has summarized over 40 years of exciting research and the development of an elegant theory. He has also reviewed recent applications of frustration theory in such areas as fetal alcohol syndrome and attention deficit-hyperactivity disorders. In this invited commentary, we briefly summarize a mathematical model of frustration theory (called DMOD) and review simulations of the model that highlight the importance of the assumptions based on frustration theory (e.g., aversiveness of unexpected nonreward, counterconditioning). We also review assumptions (e.g., unlearning, passive and active "inhibition," decline in aversiveness of expected nonreward) that are required if one is to simulate intuitive and counterintuitive phenomena.
\end{abstract}

Unfortunately, we are not always rewarded. How we learn to deal with aversive nonreward has a major impact on our success and happiness. The field of psychology has been very fortunate to include the work of Abram Amsel, who has provided us with a large database that shows the importance of nonreward. He has also developed a comprehensive theory that integrates the research results and gives us the power to make predictions. His book Frustration Theory: An Analysis of Dispositional Learning and Memory (1992), which we have been asked to comment on, is a beautiful summary of over 40 years of research and theory development. The book begins with a summary of behavioral consequences of nonreward in adult and immature rats and then reviews the effects of brain lesions and in utero exposure to alcohol. The book ends with applications of frustration theory to children with attention deficit-hyperactivity disorders.

In this commentary, we briefly review a mathematical model of frustration theory (called DMOD) and describe results of simulations that highlight the importance of nonreward as well as the importance of reintroduction of rewards.

\section{Background}

All of us have experienced nonreward. For some of us, it may have been caused by a candy machine that did not deliver our candy in return for our money, for others it may have been a lower than expected grade in a course, or an expected job offer that did not occur. Amsel has called the emotional reaction we have to this type of event frustration. A psychologist who had expressed skepti-

Correspondence should be addressed to H. B. Daly, Department of Psychology, State University of New York, Oswego, NY 13126 (e-mail: hdaly@oswego.edu). cism about the usefulness of the concept of frustration came to me one day and said,

I now know what you mean by frustration. A granting agency called me yesterday to tell me that they had approved and funded my grant, and today they called back to tell me they had run out of money just before my name.

People agree that situations that arouse frustration are unpleasant. But are these experiences helpful in some cases? Amsel's research has shown us the importance of nonreward: Prior experiences with unexpected nonreward result in persistence, "a tendency for organisms to pursue goal-directed activities despite nonreinforcement, punishment, obstacles, or deterrents - in general, in the face of any kind of negative indication" (Amsel, 1992, p. 54). However, persistence develops only if rewards are reintroduced following experiences with nonreward. We recently found an advertisement titled, "The Art of Success and Leadership! Motivational Messages." The first message listed was titled "Persistence," and the following quotation was used on the plaque:

Nothing in the world can take the place of persistence. Talent will not; nothing is more common than unsuccessful men with talent. Genius will not; unrewarded genius is almost a proverb. Education will not; the world is full of educated derelicts. Persistence and determination alone are omnipotent. (Calvin Coolidge)

If persistence is omnipotent, it would be important to determine under what conditions it develops. Amsel has given us the answer. When rats are very young (11 days or younger) they are persistent: They continue to respond when rewards are removed (extinction). They do not appear to be able to learn about the aversiveness of nonreward. But as they grow older (12 days or older), they can learn about aversive nonreward and they fail to persist. Older rats, however, show persistence if they have 
been given experience with intermittent receipt of rewards (i.e., partial reinforcement): During extinction training they persist much longer than subjects that have always been rewarded. According to Amsel, the receipt of a reward when nonreward is expected results in counterconditioning - when the approach response becomes conditioned to the stimuli of frustrative nonreward (Amsel, 1994, Figure 2).

\section{Frustration Theory and DMOD}

In his précis Amsel (1994) states,

Although my theorizing in this book alludes to mechanisms like anticipation and expectancy, which can obviously be characterized as cognitive, the constructs are those associated with neobehaviorism. I have argued that this approach is at the same time more analytical and more constraining than the more mentalistic cognitive approaches, and that a little constraint goes a long way in theorizing about behavior and its determinants (p. 281).

We have argued (Daly \& Daly, 1991) that once enough data have been collected and a comprehensive verbal model such as Amsel's frustration theory has been developed, the next logical step in constraint of a model is to put it in mathematical form and run computer simulations to determine predictions of the model. Mathematical/computer simulation models have many advantages. We (Daly \& Daly, 1991, pp. 166-167) argue that they (1) force specification of all assumptions; (2) allow one to test the importance of assumptions; (3) prevent one from overlooking assumptions; (4) aid in logical deduction and allow one to make complex and counterintuitive predictions; (5) give one an understanding of the interplay among elements of the model; (6) can prevent disagreements over language; (7) make it easier to communicate with and transfer knowledge to other fields that also use the universal language of mathematics; and (8) can show mathematical similarities between verbal models that appear different.

In 1982, we published the basic assumptions and results of over 30 successful simulations of a mathemati$\mathrm{cal} /$ computer simulation model that incorporated the major assumptions of Amsel's frustration theory (called DMOD, for Daly modification of the Rescorla/Wagner model). In 1984, we published the computer program (Daly \& Daly, 1984) and have recently made available a menu-based version of the program with an instruction manual (available from the authors). In 1987, we published an extension of DMOD that applies to aversive conditioning (e.g., electric shocks). Simulations of over 60 different learning phenomena have now been published (see Daly, 1985, 1989, 1991a, 1992b; Daly \& Daly, 1987, 1991).

In line with many other theories, we use the delta rule (linear operator) to calculate trial-by-trial changes in learning (also called the least means square [LMS] or Widrow-Hoff rule). However, we incorporate Rescorla and Wagner's (1972) assumption that learning to all stimuli present is used when one is calculating learning to a given stimulus (a modification made to allow for simulation of the Kamin blocking effect): $\Delta V=$ $\alpha \beta(\lambda-\bar{V}) . \Delta V$ is the change in associative strength on a given trial, and $\bar{V}$ is the total associative strength conditioned to all stimuli present. The three parameters are the following: alpha $(\alpha)$, the salience of the stimulus; beta $(\beta)$, the learning rate parameter; and lambda $(\lambda)$, the size of the goal event. The values for the three parameters are fixed by the conditions of the experiment. Stimuli are given an alpha of 1.0 unless salience of the stimulus is a manipulated variable. Beta is assumed to reflect the intensity of the goal reaction, which is determined by the drive level (a value of .15 is used if the rats are deprived to $80 \%-85 \%$ of their ad-lib weight). Lambda is 1.0 for a very large food reward (e.g., 15 pellets, $37 \mathrm{mg}$ ), 0.1 for a very small reward (e.g., 1 pellet, $37 \mathrm{mg}$ ), and 0.0 when no reward is present.

In line with Amsel's frustration theory, we assume that there are three possible goal events in appetitive conditioning: (1) food, which conditions approach $\left(V_{\text {ap }}\right) ;(2)$ omission of food when $V_{\text {ap }}$ is conditioned, which conditions avoidance or frustration $\left(V_{\mathrm{av}}\right)$; and (3) reintroduction of food when $V_{\mathrm{av}}$ is conditioned, which results in counterconditioning ( $V_{c c}$, or courage to approach a negative event). Behavior is based on the sum of the three $V$ values, each summed over all stimuli present: $V_{\mathrm{t}}=\bar{V}_{\mathrm{ap}}+\bar{V}_{\mathrm{av}}+\bar{V}_{\mathrm{cc}}$. The same formula is used to calculate changes in all three $V$ values, except that the lambda value varies. To condition $V_{a p}$, the lambda value is determined by the size of the food reward. To condition $V_{\mathrm{av}}$, the lambda value is the discrepancy between the obtained reward ( 0 on an extinction trial) and the expected reward (indexed by $\bar{V}_{\text {ap }}$ ). To condition $V_{\mathrm{cc}}$, the lambda value is the discrepancy between the amount of frustration experienced ( 0 on a rewarded trial) and the amount of frustration expected (indexed by $\bar{V}_{\text {av }}$ ). (See Daly \& Daly, 1982, 1987, 1991, for all formulas.)

On the basis of the research results of others, three additional assumptions are made: (1) The importance of a negative event is greater than that of a positive event (the lambda value for $V_{\text {av }}$ is twice the discrepancy; see Daly \& Daly, 1982, p. 446). (2) Negative but not positive $V$ values show spatial generalization (we multiply $V_{\text {av }}$ by .5 to determine the start speed measure assumed to be about $180 \mathrm{~cm}$ from the goal, and .75 to determine the run speed measure). (3) Following the lead of Rescorla and Wagner (1972), we assume that $V$ values are decreased in absolute magnitude at a slower rate than they are increased (a beta of .05 is used to decrement all $V$ values).

To date, we have attempted to simulate about 60 phenomena described by Amsel (1992), and all have been successful. In addition, we have extended the theory to account for and make counterintuitive predictions in a number of areas, such as preference for predictable reward events (Daly, 1992b), and the effects of toxic chemicals found in Great Lakes fish (Daly, 1992a). We are convinced that the success of the simulations is due to the two basic assumptions of Amsel's frustration theory: (1) Unexpected nonreward arouses an unconditioned 
aversive response (frustration) that is conditioned to stimuli present, and (2) subsequent reintroduction of rewards results in counterconditioning. We are also convinced that all successful theories will have to incorporate these assumptions.

\section{Review of Frustration Theory and DMOD Predictions}

In the remainder of this commentary, we will highlight the power of the assumption that unexpected nonreward is aversive.

Magnitude of reward extinction effects. Intuition tells us that more is better. Large rewards in acquisition result in faster running down a runway than small rewards, and it seems obvious that large rewards should keep our momentum going when all rewards are removed (extinction). But there is much research to show that the opposite result occurs: Larger rewards result in faster extinction. Amsel (1992) has stated that "more produce[s] less and less produce[s] more" (p. 141). This result, sometimes called a paradoxical reward ef$f e c t$, is neither paradoxical nor counterintuitive if one understands a fundamental assumption of Amsel's frustration theory: nonreward when one is expecting a large reward results in large amounts of frustration. The higher the frustration level, the more avoidance behavior occurs, and there is a rapid drop in approach to the goal. To simulate using DMOD, the lambda value for $V_{\text {ap }}$ is higher the larger the reward, which results in a higher asymptotic $V_{\text {ap }}$ value. The larger the $V_{\text {ap }}$ value the greater the discrepancy between what is obtained during extinction $(0)$ and what was expected $\left(\bar{V}_{\mathrm{ap}}\right)$, which results in a larger unconditioned reaction to the frustrating event [lambda for $V_{\mathrm{av}}$ is larger: $2\left(0-\bar{V}_{\text {ap }}\right)$; primary frustration]. The larger lambda value for $V_{\text {av }}$ results in more conditioned frustration $\left(V_{\mathrm{av}}\right)$, which results in a small $V_{\mathrm{t}}$ value and the prediction of rapid extinction. (Note that the formula is written so that $V_{\mathrm{av}}$ is a negative number, and therefore, when added to $V_{\text {ap }}$, it reduces the $V_{\mathrm{t}}$ value.)

Successive negative contrast effect (depression effect). It has been known for over 60 years that there is a rapid drop in approach to a goal when the reward is shifted from a large to a small reward. The drop is very dramatic. Within four to six experiences with the small reward, speed in approach to the goal is below that of a control group that is always given the small reward (called the successive negative contrast effect or depression effect; see, e.g., Daly, 1969). Frustration theory and DMOD can easily account for this result: Frustration or $V_{\text {av }}$ is conditioned when a smaller than expected reward is obtained. If a one-pellet reward is given, the lambda value for $V_{\mathrm{av}}$ is $2\left(.1-\bar{V}_{\mathrm{ap}}\right)$. Since $V_{\mathrm{ap}}$ is 1.0 at the end of acquisition with a large reward, there is a large discrepancy between the obtained and expected reward, and $V_{\mathrm{av}}$ is conditioned. When $V_{\mathrm{av}}$ is added to $V_{\mathrm{ap}}$, the $V_{\mathrm{t}}$ value drops below the .1 value and one obtains a negative contrast effect.
On the basis of the assumptions of Rescorla and Wagner's (1972) model, it is assumed that unlearning occurs, and when a smaller than expected reward is presented, not only does $V_{\mathrm{av}}$ get conditioned, but $V_{\text {ap }}$ declines slowly. Therefore, with extended training, the obtained and expected reward are not very discrepant, and the small reward is no longer frustrating. Thus, the lambda value for $V_{\mathrm{av}}$ approaches 0 , and $V_{\mathrm{av}}$ also declines in absolute value. Simulations of the model show recovery of the contrast effect, a result that has been obtained many times (e.g., Daly, 1991b). It appears to be difficult, if not impossible, to simulate recovery of the depression effect without the assumption that $V$ values can decrease in absolute value.

Successive positive contrast effect (elation effect). When subjects are shifted from a small to a large food reward, there is an increase in speed toward the goal. Maxwell, Calef, Murray, Shepard, and Norville (1976) showed us that only if subjects were previously shifted from a large to a small reward and had not yet recovered from the negative contrast effect do rats run faster than a control group that has always been given the large reward (positive contrast or elation effect). These are precisely the conditions under which DMOD simulations show an elation effect. If a large reward is reintroduced when $V_{\mathrm{av}}$ is still present (prior to recovery of the negative contrast effect), $V_{\mathrm{cc}}$ is conditioned, and $V_{\mathrm{t}}$ is based on $\bar{V}_{\text {ap }}+\bar{V}_{\text {av }}+\bar{V}_{\text {cc }} \cdot V_{\text {cc }}$ is the basis for the predicted elation effect.

Figure 1 presents DMOD simulations of both the depression and the elation effects. The two solid lines rep-

DMOD SIMULATION: DEPRESSION AND ELATION EFFECTS

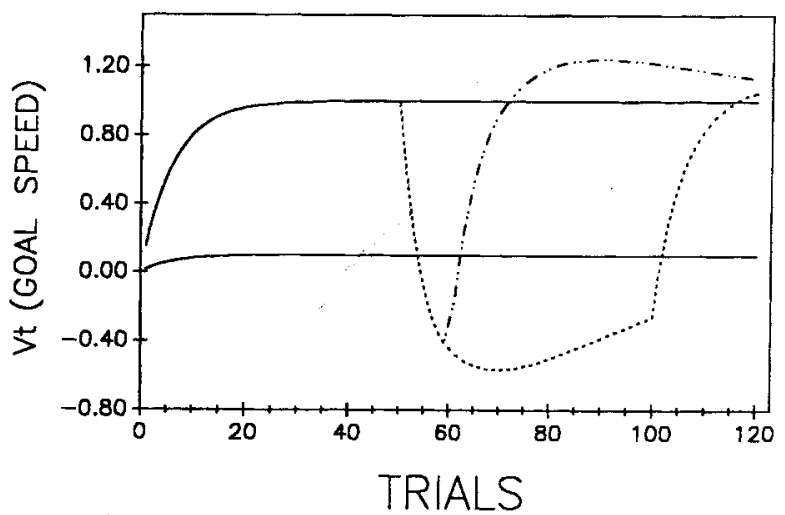

Figure 1. DMOD simulations of depression and elation effects. The upper solid line represents large reward for 120 trials; the lower solid line represents small reward for 120 trials; the dotted line represents a shift from large to small reward for $\mathbf{6 0}$ trials and shows the depression effect (slower speeds than those in the small-reward control group) and its recovery, followed by a shift to the large reward. The dashed line represents a shift from small to large reward during the depression effect and shows an elation effect (faster speed than that for the large-reward control group) and its decline with extended training. These simulations show that DMOD can make nonlinear predictions. ( $\alpha=1.0$; increment $\beta=.15$, decrement $\beta=.05, \lambda \mathrm{s}=1.0$ and 0.1 for large and small reward.) 
resent the control groups given a large food reward $(\lambda=$ $1.0)$ or a small reward $(\lambda=0.1)$. The dotted line shows the drop in speed to the goal when groups are shifted from the large to the small reward. The $V_{\mathrm{t}}$ value is below the 0.1 level of the control group, which represents the depression effect (note that negative $V_{\mathrm{t}}$ values predict approach to the goal to be lower than operant level). With extended training, the model shows recovery from the depression effect. There is no elation effect (running faster than the 1.0 level of the large-reward control group) after extended training with the small reward. The dashed line shows that DMOD predicts an elation effect if subjects are shifted to a large reward when they are still showing a depression effect. With extended training, the elation effect decreases. What is interesting about these simulations is that they show reversals in behavior (e.g., recovery of the depression effect and decrease in the size of the elation effect following extended training). These are nonlinear predictions based on a linear model. The formula used to calculate changes in the $V$ value is a linear operator. However, the assumption that there are additional important goal events (e.g., frustrative nonreward and counterconditioning following reintroduction of a large reward), based on Amsel's frustration theory, give the model some of the power of a nonlinear model.

Partial reinforcement acquisition and extinction effects. Amsel has provided a large amount of data showing that subjects on a partial reinforcement schedule (e.g., a random half of the trials are rewarded) run faster in acquisition than a group continuously reinforced when behavior is measured far from the goal (e.g., start speed measure), but slower when behavior is measured close to the goal (called the partial reinforcement acquisition effect). Both frustration theory and DMOD can account for this result. DMOD simulation results (Daly \& Daly, 1982, pp. 448-449) show that all three $V$ values are conditioned on a partial reinforcement (PRF) schedule, whereas only $V_{\text {ap }}$ is conditioned on a continuous reinforcement (CRF) schedule. Far from the goal, where $V_{\mathrm{av}}$ is assumed to have a smaller impact on behavior (spatial generalization assumption), $V_{\mathrm{cc}}$ can counteract the $V_{\text {av }}$ value and the $V_{\mathrm{t}}$ value for the PRF group is larger than that for the CRF group. Close to the goal, the $V_{a v}$ value is so large that $V_{c c}$ cannot counteract it and the $V_{t}$ value for the PRF group is smaller than that for the CRF group. This is an extremely difficult result for theories other than frustration theory or DMOD to account for. As Amsel (1992) pointed out, PRF results cannot be accounted for if one merely assumes that PRF training results in frustration tolerance, and "a more active counterconditioning mechanism seems to be required" (pp. 75-76). We have attempted to simulate many phenomena without the assumption that $V_{\mathrm{cc}}$ is conditioned, but we have been unable to simulate any result when rewards were reintroduced following conditioning of $V_{\mathrm{av}}$.

Subjects are very persistent following PRF acquisition: they continue approaching the goal even though they are never rewarded (extinction). Close to the goal, the groups show a crossover: Subjects on a PRF schedule run more slowly than subjects on a CRF schedule during acquisition but run more quickly during extinction. Within the framework of both frustration theory and DMOD, extinction following CRF training results in a large amount of frustration $\left(V_{\mathrm{av}}\right)$, which results in the rapid drop in approach to the goal. Following PRF training, there is also a high level of $V_{\mathrm{av}}$, but $V_{\mathrm{cc}}$ (counterconditioning) keeps the $V_{\mathrm{t}}$ value high and results in persistence.

Extended discrimination learning. There are at least four different sources of evidence that the stimulus associated with nonreward during learning of a discrimination is aversive (see Daly \& Daly, 1982, pp. 460-461). What is even more interesting is that aversiveness decreases with extended discrimination training. Amsel showed that the frustration effect (faster running in the second runway of a tandem runway in the presence of the stimulus associated with nonreward, $\mathrm{S}-$ ) goes away with extended training (see Amsel, 1992, p. 96). We have shown that subjects will learn a new response to escape from stimuli associated with frustrative nonreward. (We used the hurdle-jump escape response. The procedures were patterned on those used by the McAllisters to measure the aversiveness to stimuli associated with electric shocks; e.g., McAllister \& McAllister, 1971, 1991.) Following runway training, rats were placed into the goalbox of the runway and allowed to jump to a neutral box. The speed of jumping is used to index the amount of frustration aroused in the goalbox. Following limited discrimination or partial reinforcement training, both groups learned to escape from the nonreinforced goalbox, but following extended training, only the PRF group learned to escape. The group given extended discrimination training did not learn to escape, indicating that predictable nonreward following extended discrimination training is not aversive (see Daly, 1974, for a review of the escape-from-frustration research).

DMOD simulations of discrimination learning are patterned on those used by Rescorla and Wagner (1972). It is assumed that a stimulus is present on all trials (called the common cue, C; e.g., same floor texture and runway dimensions on reinforced and nonreinforced trials), a second stimulus is present only on rewarded trials $(\mathrm{S}+)$, and a third stimulus is present only on nonrewarded trials $(\mathrm{S}-)$. Simulations show that $V_{\text {ap }}$ is conditioned to the common cue on rewarded trials, which causes $V_{\mathrm{av}}$ to be conditioned to both the common cue and S- on nonrewarded trials. Therefore, DMOD can simulate both the FE and hurdle-jump escape response results following limited discrimination training. Both the conditioned $\left(V_{\mathrm{av}}\right)$ and unconditioned form (lambda for $V_{\mathrm{av}}$ or primary frustration) of aversiveness are used to index the amount of aversiveness aroused in the goalbox on an $\mathrm{S}$ - trial; and the FE and hurdle-jump speeds are faster the higher these values. If there is no unconditioned or conditioned frustration, subjects should not escape quickly from the nonreinforced goalbox.

DMOD simulations show that with extended discrimination training, the $\mathrm{S}-$ stimulus has a $-V_{\text {ap }}$ value equal 
to the $+V_{\text {ap }}$ value of the common cue (e.g., the common cue reaches a $V_{\text {ap }}$ value of +.33 , and the $\mathrm{S}$ - stimulus a value of -.33 ). Since the two add to zero, reward is not expected, and the lambda value for $V_{a v}$ is zero. Therefore, $V_{\mathrm{av}}$ approaches zero. Without the assumption that under certain conditions $V_{\text {ap }}$ can become negative and counteract the $+V_{\text {ap }}$ value of the common cue, DMOD would have no way of simulating the decrease in aversiveness of nonreward following extended discrimination training.

It should be noted that $V_{\text {ap }}$ becomes negative only under a very restricted set of circumstances in the Rescorla and Wagner (1972) model and in DMOD ( $V_{\text {ap }}$ is equivalent to the $V$ value in the Rescorla-Wagner model). This occurs when one stimulus becomes positive on a reinforced trial and is then paired with another stimulus and both are nonrewarded. The second stimulus then becomes negative, and its value grows until it is equal but opposite in sign to the first stimulus. One measures the negative properties of the second stimulus by removing the first stimulus. We have assumed that $-V_{\text {ap }}$ is passive inhibition and that its properties are different from those of $V_{\mathrm{av}}$. We assume that " $-V_{\mathrm{ap}}$ passively inhibits an approach response by subtracting from $+V_{\text {ap }}$ conditioned to other stimuli" (Daly, 1991a, p. 326). $V_{a v}$ is active "inhibition," and as Amsel (1972) has assumed, it is "a new excitatory tendency which competes with the older one" (p. 275). We assume that " $V_{\mathrm{av}}$ influences responding by making the organism attempt to actively escape or avoid cues and not just passively inhibit ongoing responses" (Daly \& Daly, 1982, pp. 444-445). (Italics added in both instances.)

Preference for predictable rewards (observing response). Since at least 1952, it has been known that if subjects are given a choice between situations where they can predict whether they will receive rewards or not and situations where rewards are unpredictable, they choose the predictable reward situation (Wyckoff, 1952). In 1974, we showed how frustration theory can account for this result. We analyzed this preference as a choice between a partial reinforcement schedule and a discrimination situation. Since we had shown that frustration does not diminish with extended partial reinforcement training but does with extended discrimination training (see earlier section), it was possible that subjects were selecting the predictable reward side to avoid the frustration on the unpredictable reward side. We used acquisition of the hurdle-jump escape response to determine the aversiveness of the stimuli associated with the predictable versus unpredictable reward situations. Only the subjects given escape training from the stimuli associated with unpredictable rewards learned the response, indicating that frustration was associated only with the unpredictable reward situation (Daly, 1974, Experiment 19; Daly \& Daly, 1991, pp. 179-182).

After simulating simple learning experiments, DMOD simulations were done on preference for predictable reward studies. No modifications or additional assumptions had to be made to successfully simulate the results.
Simulations showed that there was an underlying preference for unpredictable rewards (based on $V_{\text {ap }}$ only), and that under any conditions where unpredictable nonreward was highly aversive and $V_{\text {av }}$ was conditioned, subjects would prefer the predictable reward side. In 1992 (Daly, 1992b), we published a summary of a large body of results and showed how DMOD was able to integrate and predict the results. Variables that decrease $V_{\text {av }}$ on the unpredictable side (small rewards, no delay of reinforcement, few nonreinforced trials, alcohol) resulted in a preference for unpredictable rewards, whereas conditions that increase $V_{\mathrm{av}}$ (large rewards, long delays, many nonreinforced trials, high drive) resulted in a preference for predictable rewards (see Daly, 1985, 1989, 1992b). No other existing theory that has been applied to preference for predictable rewards is able to integrate the existing data. The basic assumptions of frustration theory combined with the power of a mathematical/ computer simulation model were able to account for all the variables that influence preference for predictable rewards (see Daly, 1992b).

Ontogeny of paradoxical reward effects. Amsel (1992) has shown that paradoxical reward effects are not present in rat pups under 12 days of age. Large continuous rewards result in slow extinction, PRF results in fast extinction, and there is no successive negative contrast effect. The paradoxical effects begin to appear around 12-14 days of age (see Amsel, chapter 7). We spent 4 exciting months with Amsel working on simulating all of the results in this area, and were able to simulate all of them with only one assumption.

Until the age of 11 days, the rat pups are assumed to operate at the level of Rescorla and Wagner's (1972) model, and only $V_{\text {ap }}$ and $-V_{\text {ap }}$ are conditioned. Rats over 11 days old are assumed to be able to learn about aversive nonreward (i.e., $V_{\mathrm{av}}$ is conditioned), and they operate at the level of DMOD.... It was assumed that young rats do not have the capacity to learn about aversive nonreward but slowly gain this ability between 12 and 26 days. One parameter reflecting the rate of conditioning of aversive nonreward, $\beta_{\mathrm{I}}$ for $V_{\mathrm{av}}$, is increased from 0 to .15 beginning at 12 days. (Daly, 1991a, pp. 325-326)

Therefore, at 11 days of age, rat pups do not condition $V_{\mathrm{av}}$, and as a result there is no $V_{\mathrm{cc}}$ conditioned. Their slow extinction reflects the slow decline in $V_{\text {ap }}$ levels. DMOD simulations indicate that when rats are rewarded on every other trial, they learn to pattern (run quickly on rewarded trials only), because $+V_{a p}$ is conditioned to the stimuli associated with nonreward, and $-V_{\text {ap }}$ is conditioned to the stimuli associated with reward. Longer intertrial intervals (ITIs) decrease the ability of the pups to pattern, but older pups are less influenced by longer ITIs. We were able to simulate the effects of age and ITI by varying the salience (alpha level) of the stimuli of reward and nonreward (lower alphas for longer ITIs) without assuming that memory varies with age. Conditioning of $V_{\mathrm{av}}$ allows older animals to pattern at longer intervals (see Daly, 1991 a, pp. 329-330). Additional research is required to determine if the assumption that 
memory for the outcome of the previous trial varies with age is necessary (Amsel, 1994).

Behavioral toxicology. Amsel's (1992) recent research has focused on variables that appear to decrease the effectiveness of frustrative nonreward: infant lesions (p. 194), prenatal exposure to ethanol (p. 197), and postnatal exposure to ethanol and $x$-radiation (p. 200). We have been focusing our research on a variable that appears to increase reactions to frustrative nonreward: the combination of toxic chemicals found in Lake Ontario salmon (Daly, Hertzler, \& Sargent, 1989; Daly, 1991b; Daly, 1992a). This, however, was not the result we expected. We used frustration theory and DMOD to guide us in our studies. After finding that adult rats fed a $30 \%$ diet of Pacific Ocean salmon from Lake Ontario for 20 days showed a larger preference for predictable rewards than did controls fed relatively uncontaminated Pacific Ocean salmon, we concluded that the toxic chemicals did not diminish learning or memory processes. Instead, these chemicals appeared to increase the aversive reaction to unpredictable nonreward ( $V_{\mathrm{av}}$ or frustration). To simulate this research, we assume that the lambda value of $V_{\mathrm{av}}$ is larger in rats fed Lake Ontario salmon. Our subsequent research showed this to be true: faster extinction and a larger successive negative contrast effect in adult rats fed Lake Ontario salmon. Offspring of rats fed Lake Ontario salmon during gestation show the same behavioral changes when tested as adults, even though they have never been fed the fish (see Daly, 1992a, for a summary).

\section{Conclusions}

Unfortunately, we are not always rewarded, and how we learn to deal with frustrative nonreward is very important. Amsel's frustration theory, along with the results of extensive research, has helped us understand the importance of nonreward and has had a major impact on psychology. Here we have been able to highlight only a small portion of his research and to consider only certain parts of the theory. We refer the reader to the 1992 book, as well as to Amsel's future papers that will review research results and applications of frustration theory. Many researchers have found Amsel's concepts to be very important. We have found frustration theory to be very helpful in understanding the behavioral effects of the combination of toxic chemicals found in Lake Ontario salmon (chemicals found all over the world). Others have found frustration theory to be helpful in therapy (e.g., Nation \& Woods, 1980). Those interested in the fetal alcohol syndrome and effects of hippocampal damage should find Amsel's recent findings fascinating. Amsel (1992) also suggests that the hyperactivity of children with attention deficit-hyperactivity disorder "reflects increased responsiveness to stimulation from anticipatory frustration" (p. 227), and that "counterconditioning of $\mathrm{s}_{\mathrm{F}} \rightarrow$ approach is, at best, slower to occur in the hyperactive child, so that the activating effects of frustration and anticipated frustration ... and the subsequent approach-avoidance conflict are not blunted" (p. 226). Research is underway to test this suggestion.
Amsel (1994) has nicely summarized the six steps he has taken in his research program (see Table 3 ), and this is a model that we have adopted: (1) Observe related behavioral effects; (2) develop a theory; (3) study the effects ontogenetically; (4) determine the neural substrates; (5) relate the prior two steps; and (6) modify the theory. Once enough data have been collected and a comprehensive verbal model has been developed, we believe that the next logical step is to put the model in mathematical form and run computer simulations to integrate the data and make intuitive and counterintuitive predictions. We have done this and found the mathematical model based on the major assumptions of Amsel's frustration theory (called DMOD) helpful in guiding our research program.

\section{REFERENCES}

AMSEL, A. (1972). Inhibition and mediation in classical, Pavlovian and instrumental conditioning. In R. Boakes \& S. Halliday (Eds.), Inhibition and learning (pp. 275-299). New York: Academic Press.

AMSEL, A. (1992). Frustration theory: An analysis of dispositional learning and memory. Canada: Cambridge University Press.

AMSEL, A. (1994). Précis of Frustration theory: An analysis of dispositional learning and memory. Psychonomic Bulletin \& Review, 1 , 280-296.

DALY, H. B. (1969). Learning of a hurdle-jump response to escape cues paired with reduced reward or frustrative nonreward. Journal of Experimental Psychology, 79, 146-157.

DALY, H. B. (1974). Reinforcing properties of escape from frustration aroused in various learning situations. In G. H. Bower (Ed.), The psychology of learning and motivation (Vol. 8, pp. 187-231). New York: Academic Press.

DaLY, H. B. (1985). Observing response acquisition: Preference for unpredictable appetitive rewards obtained under conditions predicted by DMOD. Journal of Experimental Psychology: Animal Behavior Processes, 111, 294-316.

Daly, H. B. (1989). Preference for predictable rewards occurs with high proportion of reinforced trials or alcohol injections when rewards are not delayed. Journal of Experimental Psychology: Animal Behavior Processes, 15, 3-13.

DALY, H. B. (1991a). Ontogeny of paradoxical appetitive reward effects in the rat pup: A mathematical model (DMOD) integrates results. Psychological Bulletin, 109, 325-339.

DALY, H. B. (1991b). Reward reductions found more aversive by rats fed environmentally contaminated salmon. Neurotoxicology \& Teratology, 13, 449-453.

DALY, H. B. (1992a). The evaluation of behavioral changes produced by consumption of environmentally contaminated fish. In R. Issacson \& K. F. Jensen (Eds.), The vulnerable brain: Nutrition and toxins (pp. 151-171). New York: Plenum.

DalY, H. B. (1992b). Preference for unpredictable rewards by aversive nonreward: Review of procedures, data, and theory. In I. Gormezano \& E. Wasserman (Ed.), Learning and memory: Behavioral and biological processes (pp. 80-103). Hillsdale, NJ: Erlbaum.

DALY, H. B., \& DALY, J. T. (1982). A mathematical model of reward and aversive nonreward: Its application in over 30 appetitive learning situations. Journal of Experimental Psychology: General, 111, $441-480$.

DALY, H. B., \& DALY, J. T. (1984). DMOD-A mathematical model of reward and aversive nonreward in appetitive learning situations. Program and instruction manual. Behavior Research Methods, Instruments, \& Computers, 16, 38-52.

DaLY, H. B., \& DaLY, J. T. (1987). A computer simulation/mathematical model of learning: Extension of DMOD from appetitive to aversive situations. Behavior Research Methods, Instruments, \& Computers, 19, 108-112.

DALY, H. B., \& DALY, J. T. (1991). Value of mathematical modeling of 
appetitive and aversive learning: Review and extension of DMOD. In M. R. Denny (Ed.), Aversive events and behavior (pp. 165-197). Hillsdale, NJ: Erlbaum.

DAly, H. B., HeRTZleR, D. R., \& SARGENT, D. M. (1989). Ingestion of contaminated Lake Ontario salmon by laboratory rats increases their reaction to aversive nonreward and mild shock. Behavioral Neuroscience, 103, 1356-1365.

Maxwell, F. R., Calef, R. S., Murray, D. W., Shepard, J. C., \& NoRville, R. A. (1976). Positive and negative successive contrast effects following multiple shifts in reward magnitude under high drive and immediate reinforcement. Animal Learning \& Behavior, 4, 480-484.

MCAllister, W. R., \& MCAlLister, D. E. (1971). Behavioral measurement of conditioned fear. In F. R. Brush (Ed.), Aversive conditioning and learning (pp. 105-179). New York: Academic Press.

MCAllister, W. R., \& MCAllister, D. E. (1991). Fear theory and aversively motivated behavior: Some controversial issues. In M. R. Denny (Ed.), Fear, avoidance, and phobias: A fundamental analysis (pp. 135-163). Hillsdale, NJ: Erlbaum.

Nation, J. R., \& Woods, D. J. (1980). Persistence: The role of partial reinforcement in psychotherapy. Journal of Experimental Psychology: General, 109, 175-207.

RESCORLA, R. A., \& WAGNER, A. R. (1972). A theory of Pavlovian conditioning: Variations in the effectiveness of reinforcement and nonreinforcement. In A. H. Black \& W. F. Prokasy (Eds.), Classical conditioning II: Current research and theory (pp. 64-99). New York: Appleton-Century-Crofts.

WyCKOFF, L. B. (1952). The role of observing responses in discrimination learning: I. Psychological Review, 59, 431-442.

(Manuscript received January 13, 1994: revision accepted for publication March 19, 1994.) 